\title{
Scaffolded DNA Origami of a DNA Tetrahedron Molecular Container
}

\author{
Yonggang Ke, ${ }^{\dagger}$ Jaswinder Sharma, ${ }^{\dagger}$ Minghui Liu,${ }^{\dagger}$ Kasper Jahn, ${ }^{\ddagger}$ Yan Liu,,${ }^{*} \dagger$ \\ and HaO Yan ${ }^{*, t}$
}

\begin{abstract}
Department of Chemistry and Biochemistry and The Biodesign Institute, Arizona State
University, Tempe, Arizona 85287, and Centre for DNA Nanotechnology and iNANO,

Aarhus University, Aarhus, Denmark DK-8000
\end{abstract}

Received April 10, 2009; Revised Manuscript Received April 30, 2009

\begin{abstract}
We describe a strategy of scaffolded DNA origami to design and construct 3D molecular cages of tetrahedron geometry with inside volume closed by triangular faces. Each edge of the triangular face is $\sim 54 \mathrm{~nm}$ in dimension. The estimated total external volume and the internal cavity of the triangular pyramid are about $1.8 \times 10^{-23}$ and $1.5 \times 10^{-23} \mathrm{~m}^{3}$, respectively. Correct formation of the tetrahedron DNA cage was verified by gel electrophoresis, atomic force microscopy, transmission electron microscopy, and dynamic light scattering techniques.
\end{abstract}

DNA has been considered an ideal material for building nanostructures at nanometer to micrometer scale because of its well-characterized double helical model and highly specific Watson-Crick base pairing. In recent years, a variety of one-dimensional (1D) and two-dimensional (2D) DNA nanostructures have been developed and utilized as templates for directed assembly of other materials. ${ }^{1}$ To date, a few examples of polyhedral three-dimensional (3D) DNA constructs $^{2}$ have been demonstrated through either one-step or hierarchical assembly approach, further proving that DNA nanoassembly is highly programmable. Nevertheless, these polyhedral structures are wire framed in geometry that share a common feature that rigid DNA duplex(es) serve as polyhedral wire edges and flexible branch point(s) serve as vertexes. As a result, each face of the 3D objects is open. A more sequence addressable, closed-face molecular cage is highly desirable as nanometer-sized containers to encapsulate other materials. Inspired by the recent exciting development of scaffolded DNA origami for 2D nanopatterning, ${ }^{3}$ we here describe a strategy to design and construct $3 \mathrm{D}$ molecular containers of tetrahedron geometry with inside volume closed by triangular faces.

The design of the tetrahedron DNA container is achieved by modifying the strategy used in the 2D DNA origami (Figure 1). In the 2D DNA origami, the single-stranded M13 DNA scaffold is designed to turn, and go the opposite direction, when it comes to the end point. Because this is a $3 \mathrm{D}$ structure, there is no end point to the surface. As illustrated in Figure 1c, when the M13 strand (red-colored

\footnotetext{
* To whom correspondence should be addressed. E-mail: (H.Y.) hao.yan@ asu.edu; (Y.L.) yan_liu@asu.edu.

Arizona State Ūniversity.

* Aarhus University.
}

strand) comes to the bottom right (where the number 1 is), it continues at the bottom left at the number 1 . This means that the scaffold strand runs through the entire structure without turning with the exception of the hairpin loops at the top right and bottom left of Figure 1b,c. This also eliminates any seams that would arise from making $2 \mathrm{D}$ segments, which are subsequently bridged by staple strands.

The following design features of the tetrahedron container can also be seen in Figure 1b,c: for all the rows with even numbers at the edges, the $5^{\prime}$ to $3^{\prime}$ direction of the M13 strand is running toward the left, and in all the rows with odd numbers at the edges the $5^{\prime}$ to $3^{\prime}$ direction of the M13 strand is running toward the right (the tracing of the M13 scaffold can also be seen in the Supporting Information). This makes adjacent rows antiparallel. The helices in the rows are segmented in a fashion so that they display a planar triangular face. The staple strands will fold the M13 scaffold strand into a closed tetrahedron container as illustrated in Figure 1a,d. Note that to allow the adjacent triangles to close up to form the tetrahedron geometry, a single nucleotide flexibility was used during the design, as illustrated as small single-stranded spacing between the edges of neighboring triangles. Two nucleotide's flexible spacing was used between the leftmost and the rightmost triangle faces shown in Figure 1c. Another important feature in this design is that there are two kinds of edges of the triangle faces, edges formed between parallel helices (i.e., edges connecting the top and bottom helices) and edges formed between continued helices (i.e., the other edges shown in Figure 1b,c). In our construction, we deliberately designed this to allow the same type of edges to meet to close up and form the tetrahedron cage. 


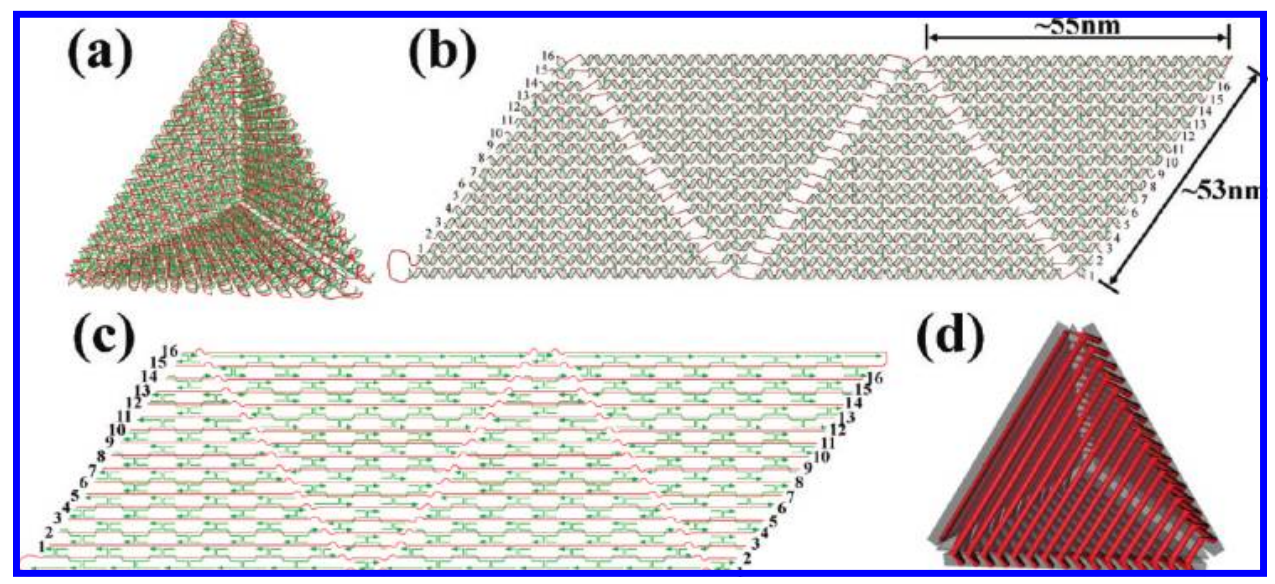

Figure 1. (a) A 3D model of a DNA tetrahedron with closed triangular faces. (b,c) A 2D model and a schematic drawing of the tetrahedron cage opened along the edges to illustrate the strand tracing. Staples (green) and circular scaffold (red) hybridize and form four triangular structures that are connected together by the consecutive scaffold. The numbers on the two sides (1 to 16) indicate the continuous connections of the scaffold. (d) A 3D schematic drawing shows the scaffold arrangement in the DNA tetrahedron.

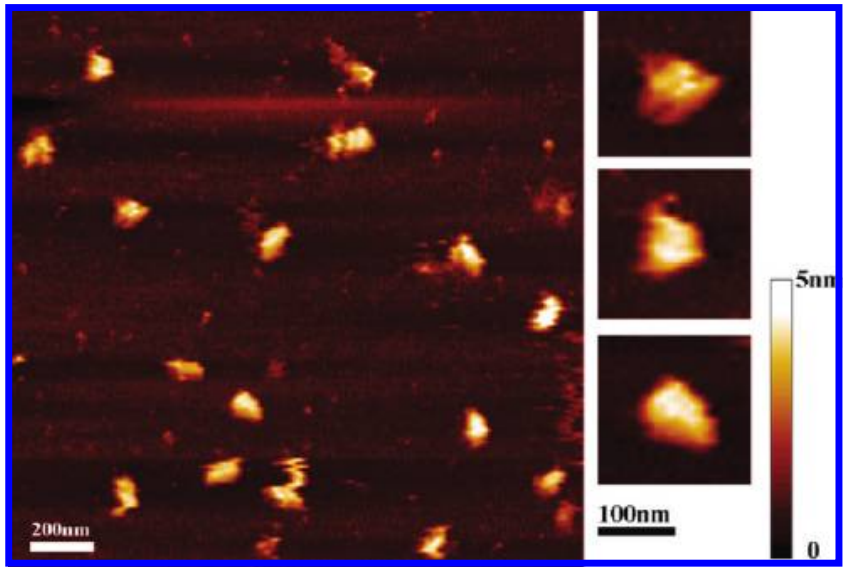

Figure 2. AFM images. The tetrahedron DNA cages collapse on mica surface, forming two-layered structures with $4-5 \mathrm{~nm}$ heights.

In our design, the M13 single-stranded scaffold DNA, consisting of 7429 base pairs, is held together by 248 short single-stranded DNA oligos (see Supporting Information, Table S1) to form the tetrahedron container. Each edge of the triangular face is $\sim 54 \mathrm{~nm}$ in dimension. The estimated total external volume and the internal cavity of the triangular pyramid are about $1.8 \times 10^{-23}$ and $1.5 \times 10^{-23} \mathrm{~m}^{3}$, respectively (see Supporting Information for detailed calculation of dimension and volume).

The DNA triangular pyramid was assembled through a single step synthesis process. All staples and the M13 scaffold were mixed together and slowly annealed from 90 to $4{ }^{\circ} \mathrm{C}$ (see Supporting Information for materials and methods). Nondenaturing agarose gel electrophoresis (Supporting Information, Figure S1) showed a distinct band that suggests homogeneous DNA structures were formed after the annealing process. AFM was further used to verify the discrete objects (Figure 2). The $x-y$ dimension revealed that all the structures have consistent size. The dimension appears to be larger than the expected $\sim 54 \mathrm{~nm}$ size of the tetradedron edge due to the lower lateral resolution of AFM imaging and possible distortion of the structure by AFM cantilever that caused the structure to collapse. Nevertheless, zooming in on some of the less distorted structures shows a triangularshaped geometry in the $x-y$ dimension, indicating the correct formation of the structure. However, because of the nanostructures being distorted by the AFM tip the evidence of the triangular structure from AFM on the assembled particles is not definitive. The measurement of the $z$ direction shows a height of $\sim 4-5 \mathrm{~nm}$, corresponding to the height of a collapsed tetrahedron cage to form a two-layered DNA structure. Although the structures are distorted during tip scanning across the surface, AFM imaging combined with gel shift assays clearly shows that discrete objects of monodisperse sizes were formed when applying the designed strategy. In addition, AFM images of the partial structure of the DNA tetrahedron formed by removal of the staple strands for two entire faces shows a single layer parallelogram formation, which further proves the folding strategy of the designed structure (Supporting Information,Figure S4).

To further reveal the structural conformation of the tetrahedron cage, transmission electron microscopy (TEM), a less destructive imaging technique for 3D DNA structures, was used to characterize the sample. The sample solution was dropped on a carbon-coated grid and negatively stained with uranyl formate. Individual DNA particles are clearly visible in the images (Figure 3) and high-resolution TEM revealed that nearly $40 \%$ of those particles adopt the designed tetrahedron shape. There are also particles showing distorted or broken shapes. This is likely due to the damage caused by the staining process. The edges of those tetrahedron-like particles show different contrast, as a result of the higher density of DNA at the edges. Additional high-resolution TEM images are shown in the Supporting Information (Figures S2 and S3).

We further performed a DLS experiment to assess the distribution of particle sizes in solution (Figure 4). The measurements were done in $300 \mu \mathrm{L}$ at a $2 \mathrm{nM}$ concentration in three runs. As can be seen, the size distribution of each run looks very reproducible. From the three runs, we obtained a value for the peak of the curve of $41.4 \pm 2.1 \mathrm{~nm}$ and a 


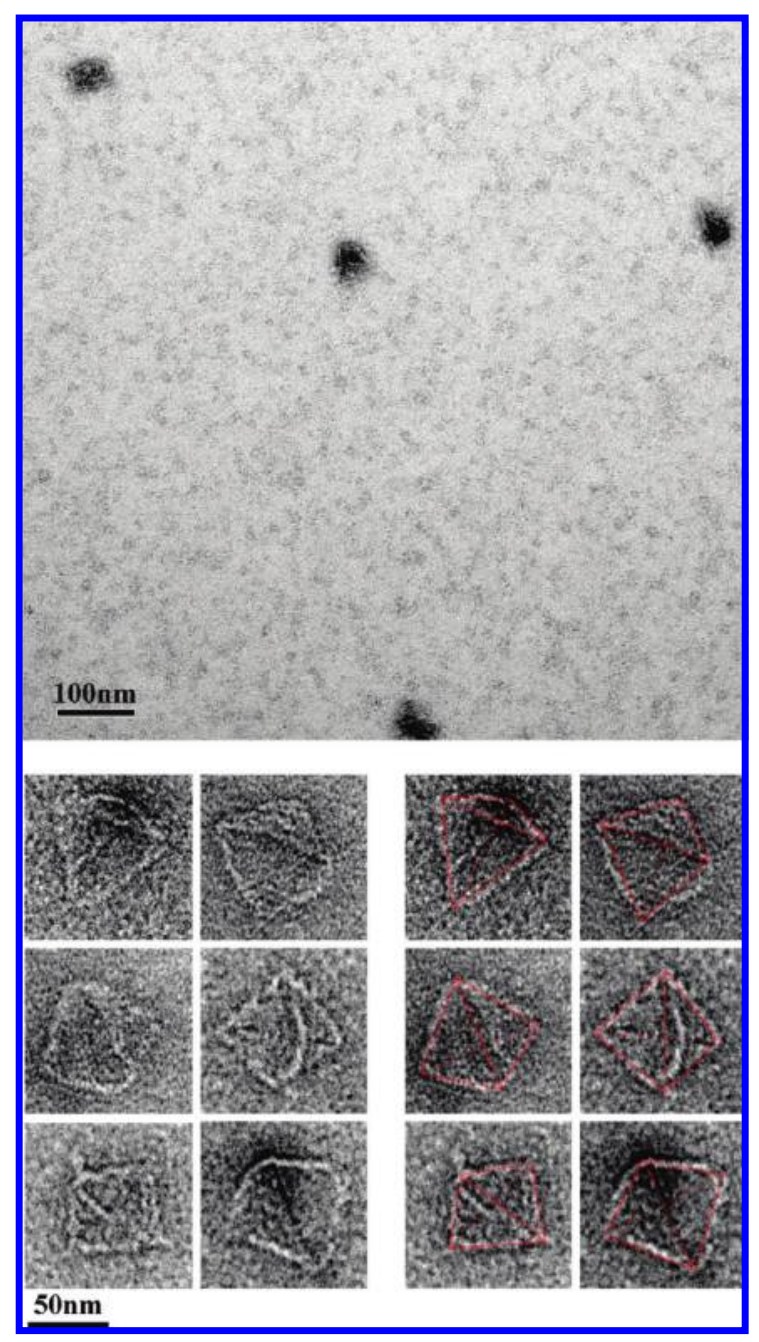

Figure 3. TEM images. Top: zoom-out images. Bottom: zoom-in images and images superimposed with the tetrahedron geometry. The edges of the DNA tetrahedron cages $(\sim 54 \mathrm{~nm})$ are clearly visible.

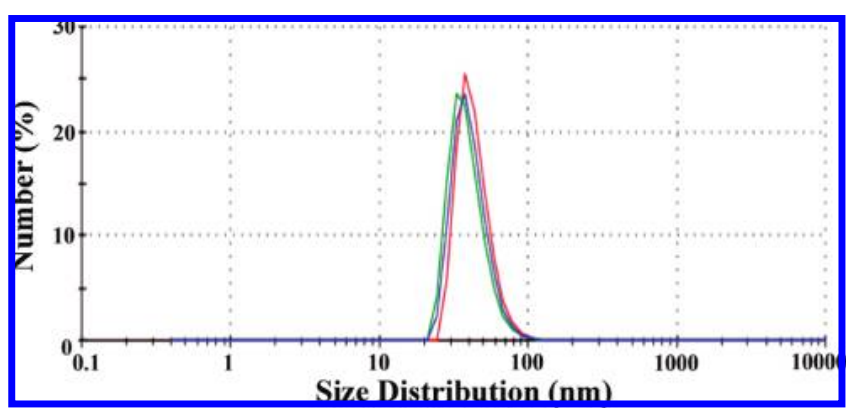

Figure 4. Diameter size distribution of the DNA tetrahedron cages from DLS measurements. The three different colored (red, green, and blue) lines represent the three runs.

value for the width of the curves of $11.7 \pm 0.3 \mathrm{~nm}$. This value fits well compared to the theoretical value for the hydrodynamic radius of the DNA tetrahedron container of $24.6 \mathrm{~nm}$ which gives a diameter of $49.2 \mathrm{~nm}$.

It is noted that the tetrahedron container structure is likely to be a mixture of two different isomers, one presenting one face of the origami to the outside and one presenting a different face. Further studies such as cryo-EM imaging could be used to characterize such properties as well as the $3 \mathrm{D}$ conformation of the structures. It would also be interesting to design additional features such as protruding hairpin loops on selective side of the triangular faces to induce the folding of the origami structure into DNA tetrahedrons of a chosen chirality.

In summary, we have successfully designed, assembled, and characterized a closed DNA tetrahedron with an inside cavity, much resembling the icosahedral structure of many virus particles. In principle, the same design strategy could be used to construct other DNA-based molecular cages as well. The next obvious challenge is to encapsulate cargos (e.g., proteins, metallic nanoparticles, virus genes, and capsids) inside the DNA cage. Such cargo-vector assembly could be used for nanoparticle site-specific functionalization or target-specific delivery in which the DNA structure is labeled with target recognition tags outside it. This could be done because each of the 248 staple strands represent an addressable point of the surface, which can be modified with various chemistries to have different properties. One could also envision protecting an enzyme from proteases with the container, and at the same time allowing the encapsulated protein to be active, since small substrate/product molecules can access the container through the small holes in the DNA mesh.

Acknowledgment. This work has been supported by grants from NSF, NIH, ARO, ONR, and AFOSR to H.Y. and from ARO and NSF to Y.L. H.Y. is also supported by the Sloan Research Fellowship. We would like to thank Ebbe S. Andersen and Jørgen Kjems of Aarhus University for their help with the manuscript.

Supporting Information Available: DNA sequences, experimental methods, Agarose Gel, additional TEM images. This material is available free of charge via the Internet at http://pubs.acs.org.

\section{References}

(1) (a) Seeman, N. C. Nature 2003, 421, 427-431. (b) Aldaye, F. A.; Palmer, A. L.; Sleiman, H. F. Science 2008, 321 (5897), 1795-1799. (c) Lin, C.; Liu, Y.; Yan, H. Biochemistry 2009, 48 (8), 1663-1674.

(2) (a) Chen, J.; Seeman, N. C. Nature 1991, 350, 631-633. (b) Zhang, Y.; Seeman, N. C. J. Am. Chem. Soc. 1994, 116, 1661-1669. (c) Shih, W. M.; Quispe, J. D.; Joyce, G. F. Nature 2004, 427, 618-621. (d) Goodman, R. P.; et al. Science 2005, 310, 1661-1665. (e) Erben, C. M.; Goodman, R. P.; Turberfield, A. J. J. Am. Chem. Soc. 2007, 129, 69926993. (f) He, Y. Nature 2008, 452, 198-201. (g) Aldaye, F. A.; Sleiman, H. F. J. Am. Chem. Soc. 2009, 129, 13376-13377. (h) Bhatia, D.; Mehtab, S.; Krishnan, R.; Indi, S. S.; Basu, A.; Krishnan, Y. Angew. Chem., Int. Ed. 2009, 48 [Online early access]. DOI: 10.1002/ange. 200806000. (i) Ghodke, H. B.; Krishnan, R.; Vignesh, K.; Kumar, G. V. P.; Narayana, C.; Krishnan, Y. Angew. Chem. Int. Ed. 2007, 46, 2646-2649. (j) Mastroianni, A. J.; Claridge, S. A.; Alivisatos, P. A. J. Am. Chem. Soc. [Online early access]. DOI: 10.1021/ja808570g.

(3) Rothemund, P. W. K. Nature 2006, 440, 297-302.

NL901165F 